\title{
Hubble Space Telescope Observations of M32: The Color-Magnitude Diagram"
}

\author{
Carl J. Grillmair \\ Jet Propulsion Laboratory, California Institute of Technology, 4800 Oak Grove Drive, Pasadena, \\ California 91109-8099 \\ Tod R. Lauer \\ Kitt Peak National Observatory, National Optical Astronomy Observatories,2] P.O. Box 26732, \\ Tucson, Arizona 85726 \\ Guy Worthey ${ }^{3}$ \\ Department of Astronomy, University of Michigan, Ann Arbor, Michigan 48109-1090 \\ S. M. Faber \\ UCO/Lick Observatory, Board of Studies in Astronomy and Astrophysics, University of \\ California, Santa Cruz, California 95064 \\ Wendy L. Freedman \\ The Observatories of the Carnegie Institution of Washington, 813 Santa Barbara Street, \\ Pasadena, California 91101 \\ Barry F. Madore \\ NASA/IPAC Extragalactic Database, Infrared Processing and Analysis Center, California \\ Institute of Technology, Pasadena, California 91125 \\ Edward A. Ajhar \\ KPNO, National Optical Astronomy Observatories, ${ }^{2}$ P.O Box 26732, Tucson, Arizona 85726 \\ William A. Baum \\ Department of Astronomy, Box 351580, University of Washington, Seattle, Washington 98195 \\ Jon A. Holtzman \\ Astronomy Department, New Mexico State University, Box 30001 / Dept. 4500, Las Cruces, New \\ Mexico 88003 \\ C. Roger Lynds
}

Kitt Peak National Observatory, National Optical Astronomy Observatories, ${ }^{2}$ P.O. Box 26732, Tucson, Arizona 85726

\footnotetext{
${ }^{1}$ Based on observations with the NASA/ESA Hubble Space Telescope, obtained at the Space Telescope Science Institute, which is operated by AURA, Inc., under NASA contract NAS 5-26555.

${ }^{2}$ Operated by AURA, Inc., under cooperative agreement with the National Science Foundation

${ }^{3}$ Hubble Fellow
} 
Earl J. O’Neil, Jr.

Kitt Peak National Observatory, National Optical Astronomy Observatories, ${ }^{2}$ P.O. Box 26732, Tucson, Arizona 85726

Peter B. Stetson

Dominion Astrophysical Observatory, 5071 W. Saanich Rd., RR5, Victoria, British Columbia V8X 4M6, Canada

\begin{abstract}
We present a $V-I$ color-magnitude diagram for a region $1^{\prime}-2^{\prime}$ from the center of M32 based on Hubble Space Telescope WFPC2 images. The broad color-luminosity distribution of red giants shows that the stellar population comprises stars with a wide range in metallicity. This distribution cannot be explained by a spread in age. The blue side of the giant branch rises to $M_{I} \approx-4.0$ and can be fitted with isochrones having $[\mathrm{Fe} / \mathrm{H}] \approx-1.5$. The red side consists of a heavily populated and dominant sequence that tops out at $M_{I} \approx-3.2$, and extends beyond $V-I=4$. This sequence can be fitted with isochrones with $-0.2<[\mathrm{Fe} / \mathrm{H}]<+0.1$, for ages running from 15 Gyr to 5 Gyr respectively. We do not find the optically bright asymptotic giant branch stars seen in previous ground-based work and argue that the majority of them were artifacts of crowding. Our results are consistent with the presence of the infrared-luminous giants found in ground-based studies, though their existence cannot be directly confirmed by our data. The tip of the metal-poor portion of the giant branch occurs at the luminosity expected if M32 is at the same distance as M31 but is too sparsely sampled by this data set to provide a precise distance estimate. At fainter magnitudes, the rising giant branch is significantly wider $\left(\mathrm{FWHM}_{V-I} \sim 0.6 \mathrm{mag}\right.$ down to $\left.M_{I} \sim-1.0\right)$ than can be accounted for by photometric uncertainties, again due to a metallicity spread. There is little evidence for an extended or even a red horizontal branch, but we find a strong clump on the giant branch itself, as expected for the high metallicities inferred from the giant branch. If the age spread is not extreme, the distribution of metallicities in M32 is considerably narrower than that of the closed-box model of chemical evolution, and also appears somewhat narrower than that of the solar neighborhood. Overall, the M32 $H S T$ color-magnitude diagram is consistent with the average luminosity-weighted age of $8.5 \mathrm{Gyr}$ and $[\mathrm{Fe} / \mathrm{H}] \approx-0.25$ inferred from integrated spectral indices, extrapolated to the same radius and analyzed with the same population models.
\end{abstract}

Subject headings: galaxies: abundances, galaxies: elliptical and lenticular, galaxies: evolution, galaxies: individual: NGC 221, galaxies: Local Group 


\section{Introduction.}

As the nearest example of an elliptical galaxy, M32 serves as an important beachhead in our campaign to understand the age and metallicity mixture of stellar populations in elliptical galaxies. While M32 may be somewhat unusual given its proximity to M31, spectroscopically it is identical to other faint ellipticals (Faber 1972; Burstein et al. 1984; Bica, Alloin, \& Schmidt 1990; González 1993; Trager et al. 1996). A key objective is to obtain deep color-magnitude (CM) diagrams to test conclusions thus far based solely on integrated colors and spectral index work. The proximity of M32, combined with the superb resolution afforded by the Hubble Space Telescope Wide Field/Planetary Camera 2 (WFPC2) permits a remarkable improvement in our ability to study its stellar content and brings us within range of this goal.

In studying a stellar population, the basic variables we wish to determine are the mean stellar age and metallicity, and the distribution functions around these meansf. For old stellar populations like that in M32, it is difficult to measure both age and $\mathrm{Z}$ simultaneously from integrated light, as broad-band spectral shape and metal lines tend to vary together in lock step with both these variables (Worthey 1994). To break this "age-Z degeneracy", it is necessary to add another measured quantity that is specifically sensitive to age. Four such quantities have been suggested for M32: 1) Balmer line strength (Burstein et al. 1984, Faber et al. 1992), which is directly sensitive to turnoff temperature, 2) Sr II $\lambda 4077 / \mathrm{Fe}$ I $\lambda 4045$ (Rose 1985, 1994), which is sensitive to the ratio of dwarf to giant light and hence to turnoff luminosity, 3) ultraviolet surface-brightness fluctuations at $2500 \AA$ (Worthey 1993), again sensitive to turnoff luminosity, and 4) (1500-V) color (Bressan, Chiosi, \& Tantalo 1996), which measures age through its effect on the number and luminosities of hot post-RGB stars.

Many integrated light studies have claimed to have found evidence for a relatively young, few-Gyr-old stellar population in M32 (e.g. Baum 1959, Faber 1972, O'Connell 1980, Burstein et al. 1984, Rose 1985, Bica, Alloin \& Schmidt 1990, Hardy et al. 1994). However, in retrospect it is clear that some of these investigations lacked the basic spectral information needed to distinguish age and Z, while others lacked sufficiently reliable population models to correctly interpret the important spectral features.

Rapid progress is being made on both fronts. Stellar population models, taking both age and Z into account, have been constructed by Worthey (1994), Bressan et al. (1994), Buzzoni (1995), and by Tantalo et al. (1996). Accurate measurements of $\mathrm{H} \beta$ (Gonzáles 1993, hereafter G93) and $\mathrm{Sr} / \mathrm{Fe}$ (Rose 1994) in M32 are also now available (the former as a function of radius out to $1 \mathrm{R}_{e}$ ). The upshot of this recent work is to confirm the earlier findings of relatively young stars, but the conclusion is now considerably more secure.

Nevertheless, there are important assumptions in these population models that need to be

\footnotetext{
${ }^{4}$ For some elliptical populations, the Fe-peak/ $\alpha$-element ratio also appears to vary (Worthey Faber, \& Gonzáles 1992), but fortunately M32's element ratios seem close to solar, with Fe/Mg perhaps just slightly enhanced.
} 
checked against a real CM diagram. For example, Worthey's models for the age and Z of M32 assume a pure red clump for the horizontal branch (HB). If the $\mathrm{H} \beta$ were concentrated at intermediate F-type temperatures, the need for a young population would be completely removed (Burstein et al. 1984). All models also assume a luminosity function for RGB and AGB stars that crucially affects the predicted red and infrared continua, spectral features, and surface-brightness fluctuations. Finally, the CM diagram permits measurement of the spreads about mean properties in a way that integrated light can never do. These spreads are as important as the mean values in decoding the star-formation history of the galaxy.

This paper uses a new upper CM diagram of M32 from HST to address these and other fundamental aspects of the stellar population. The major focus is on the metallicity and its distribution. That is because giant-branch loci are much more sensitive to $\mathrm{Z}$ than age, so conclusions about $\mathrm{Z}$ are relatively independent of the assumed age. The breadth of the giant branch implies a wide range in $\mathrm{Z}$, from roughly solar down to below -1.0 dex. Paradoxically though, the distribution is rather narrow when judged by its FWHM compared to either the closed-box model of chemical enrichment or the distribution of metallicity in the solar neighborhood. Evidence for a wide giant branch and broad total range in $\mathrm{Z}$ was available before from pioneering ground-based CM diagrams in $V$ and $I$ by Freedman (1989) and Davidge \& Jones (1992). However, it is clear now that the old data were just the tip of the iceberg - the present data give a much fuller picture of the upper giant branch, and a correspondingly greater total width in $V-I$.

A second focus is comparison with mean population age and $\mathrm{Z}$ deduced from ground-based integrated spectral indices. We can infer these values using Worthey models for the HST field by slight outward extrapolation of G93's data. The resulting age-Z pair, 8.5 Gyr and -0.25 dex, agrees perfectly with the color of the giant-branch stars in the CM diagram. This indicates a degree of consistency in the Worthey models but does not independently establish the age. Ages could be inferred from very deep observations of main sequence turnoff stars, but crowding precludes this in M32 inside about 3 arcmin, even with HST. Since upper giant-branch tracks are degenerate in age and $\mathrm{Z}$ much like integrated colors and metallic lines, it is not possible to derive an age from the upper CM diagram alone. Aside from verifying the red clump assumption of the Worthey models (in itself an important contribution), the new CM diagram has little to say about age.

Nonetheless, the new observations represent an enormous gain over previous ground-based

efforts in our ability to probe the stellar population of M32. They offer another tantalizing glimpse of how this so very interesting galaxy may have formed, and point the way for future work.

\section{Observations.}

Images of M32 were taken on October 22, 1994, with WFPC2. Two fields were imaged, one of M32 (POS1) and the other of a "background" field (POS2) to determine the colors and magnitudes of the M31 stars that make up the majority of field stars at POS1. The POS1 coordinates were 
chosen so that the nucleus of M32 was roughly centered in WF3. The Planetary Camera (PC) image ( 0 " 0455 per pixel) covers a region extending from 1 to 2 arcminutes almost due south of the center of M32 (see Figure 1 = Plate 1). The POS2 field is situated $\approx 5.6 \mathrm{NE}$ of POS1 in an area originally intended to match the background surface brightness of M31 underlying M32 f. Four 500s exposures were taken in each of F555W $(V)$ and F814W $(I)$ in each pointing. An additional three 1000s exposures were taken through F300W, but we defer discussion of these data to a later paper. The coadded F814W image is shown in Figure 2 (Plate 2).

Stellar photometry was carried out using the crowded-field, PSF-fitting package ALLFRAME (Stetson 1994), the most recent development in the DAOPHOT series of photometry packages. ALLFRAME differs from its predecessors primarily in its ability to use information from many individual frames simultaneously. The extra geometric and photometric information available from multiple frames extends the range of magnitudes and crowding conditions for which useful photometry is obtainable. ALLFRAME is applied to the data after object detection and aperture photometry are carried out using DAOPHOT II (Stetson 1987). The global characteristics of the CM diagram produced using DAOPHOT II and ALLSTAR were essentially identical to those found using ALLFRAME. However, the latter produced fewer outliers and gave a cleaner-looking result.

To push the star detection limit to as faint a level as possible, we coadded all images taken through the same filter. ALLFRAME was then applied to the F555W and F814W images simultaneously. While analyzing the coadded images does not make full use of the information available in all 8 individual frames, experiments using both methods showed that our completeness fraction was significantly higher when coadding frames prior to object detection.

Neither POS1 nor POS2 had a sufficient number of bright and reasonably isolated stars to allow proper characterization of the PSF. The PSF was consequently derived from F555W and F814W images of the globular cluster NGC 6397 observed with HST approximately two months prior to the date of the M32 observations. No focus changes were commanded during the intervening period, and desorption of volatiles will not have significantly altered the focus over this time.

Owing to severe crowding, we ran three DAOPHOT II detection passes to identify and measure faint stars and to improve the photometry for the bright stars. After each pass, we subtracted all detected stars from the images and reran the finding algorithm to search for any newly-revealed faint stars. Three such passes yielded a candidate list of roughly 24,000 objects. Passing this list on to ALLFRAME resulted in the elimination of $\approx 4000$ objects, leaving a total of 19,969 measured stars. The final CM diagram is approximately one magnitude deeper at the $50 \%$ completeness level than it had been using a single detection pass.

Identical procedures were used to find and measure stars in the POS2 field, yielding a total of 4111 stars. Under ideal conditions, correcting for M31 background stars in the M32 field would require that, for each star found in POS2, we subtract from the POS1 sample the star most similar

\footnotetext{
${ }^{5}$ Due to a pointing error, this match was not quite achieved — see below.
} 
in color and magnitude. However, image crowding is clearly more important for POS1 than POS2, and proper accounting needs to be taken of their differing levels of completeness. Moreover, owing to a miscalculation, the POS2 field as observed lies about 57" outwards from the M31 isophote that passes through POS1. The number of M31 stars we see in POS2 consequently underestimates the number we would expect to find in POS1. From interpolation of blue M31 isophotes plotted by Hodge \& Kennicutt (1982) and by deVaucouleurs (1958) and from color-index data by Walterbos \& Kennicutt (1988), we estimate the $V$ surface brightness gradient of M31 in the vicinity of M32 to be approximately $0.0035 \mathrm{mag} / \mathrm{arcsec}$. From this we conclude that the stellar surface density expected at POS1 is 1.2 times that found at POS2.

Completeness tests were carried out by adding $\approx 500$ artificial stars, with colors and magnitudes selected from grid points in the CM diagram, to each of the POS1 F555W and F814W images. The pixel locations of the added stars were randomly chosen and identical between F555W and F814W frames. The frames were then processed using DAOPHOT II and ALLFRAME in a manner identical to that applied to the original data. The results of these tests are shown in Figures 3 and 4 , which show that the $50 \%$ completeness level for most POS1 stars occurs at $I \approx 24.9$ and $V \approx 25.4$. Virtually identical tests were carried out for POS2.

The M32 CM diagram was corrected for contaminating M31 stars as follows. For a star at POS2 of magnitude $I$ and color $V-I$, a number of stars

$$
N=1.2 \times C_{1}(I, V-I) / C_{2}(I, V-I)
$$

of similar magnitude and color were subtracted from the POS1 sample, where $C_{1}$ and $C_{2}$ denote completeness fractions computed at POS1 and POS2, respectively, and the factor 1.2 corrects for the misplacement of POS2. In most cases $N$ is not an integer, and stars were subtracted once, twice, or not at all as determined by whether a randomly generated number fell above or below the fractional portion of $N$. In total, about 3600 stars were subtracted from the POS1 sample to account for contamination by M31.

The completeness tests also showed that the level of crowding leads to relatively large photometric uncertainties, ranging from $\pm 0.19 \mathrm{mag} R M S$ at $I=22.0$ to \pm 0.46 mag RMS at $I=25.0$. However, the uncertainties in $V$ and $I$ are correlated, and the color uncertainties range from \pm 0.08

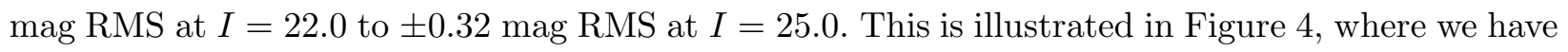
plotted the colors and magnitudes of artificial stars as returned by the DAOPHOT/ALLFRAME processing sequence.

\section{Discussion}




\subsection{Morphology of the Color-Magnitude Diagram}

The CM diagrams for the POS1 and POS2 fields are shown in Figures 5 and 6. For stars bluer than $V-I=3$ the magnitudes have been transformed from F555W and F814W to Johnson-Cousins $V$ and $I$ using the coefficients of Holtzman et al. (1995). The $V-I$ color terms determined by Holtzman et al. are poorly constrained for very red stars. They are approximately zero at $V-I=$ 2.5 (the reddest color for which good measurements exist) and we have consequently adopted zero color terms for all stars with $V-I>3.0$. The magnitudes have also been dereddened assuming $\mathrm{E}(B$ $V)=0.08$ (Burstein \& Heiles 1982) and using the absorptions tabulated by Holtzman et al. (1995) for stars of K5 spectral type. An electronic version of the photometry table is available on request from CJG.

The most striking feature in Figure 5 is the obviously composite nature of the CM diagram there is a wide spread in the colors of the giants. Particularly interesting is the strongly enhanced red sequence that bends over at $I \approx 21.2$, and extends to extremely red $(V-I>4)$ colors. While Freedman (1989) and Davidge \& Jones (1992) showed that the giant branch covers a wide range in color, this extended sequence was not visible in either previous work and represents a significant change in the picture of the M32 RGB. The present CM diagram is consistent, however, with Freedman's noted incompleteness at faint $V$ magnitudes and her corresponding insensitivity to very red stars. This insensitivity is obvious when one considers the effect of a $V$-magnitude limit given the morphology apparent in the $V$ vs $V-I$ diagram, illustrated in Figure 6 . In short, the new HST measurements reveal the full extent and morphology of the upper giant branch for the first time. Even with HST, however, there may be some incompleteness for very red stars with $V-I>3$. For example, we find 14 starlike objects brighter than $I=22$ that have no visible counterparts at all in the $\mathrm{F} 555 \mathrm{~W}$ image. Most of these are probably extremely red giants with $V-I>4)$.

Below about $I=22$, the giant stars appear to merge into a rather fat giant branch of more or less uniform width down almost to the clump. Based on the completeness tests, the width of the giant branch above $I \sim 24$ is significantly larger than can be accounted for by photometric errors (the color uncertainty at $I=23$ is $0.125 \mathrm{mag}$ RMS, whereas the observed giant branch width at this point is $0.253 \mathrm{mag}$ RMS — we return to this point below). The core-helium burning stars seem to be concentrated in a red clump. We see no indications of RR Lyraes or an extended reddish horizontal branch, even though the completeness in the region of the diagram where they should appear $(I \sim 24.6, V-I \sim 0.5)$ is $\sim 65 \%$. The form of the fall-off in the density of stars below $I=25$ is due to a combination of completeness effects and photometric uncertainties.

\subsection{Crowding and the Brightest Giants}

We observe a significant number of stars to be brighter than the first ascent RGB tip. These may be AGB stars. However, their luminosities are significantly fainter than the AGB stars iden- 
tified by Freedman (1989). Assuming a distance modulus $(m-M)_{0}=24.43$ (Ajhar et al. 1996), we expect the tip of the giant branch for older/more metal-poor populations to occur at $I \approx 20.4$. We find 23 stars with $I<20.4$ in POS1 and only one such star in POS2, giving a surface density of AGB stars of $70 \mathrm{arcmin}^{-2}$. Though the absence of such stars in POS2 argues that these stars must be associated with M32, they do not appear to be as strongly concentrated towards the center of M32 as the integrated light. Freedman finds 91 stars brighter than $I=20.4$ in her inner field (a $1.42 \operatorname{arcmin}^{2}$ region of her field which encompasses POS1 - see Figure 1), giving a very similar surface density of $64 \operatorname{arcmin}^{-2}$.

However, whereas our $I$-band luminosity function goes essentially to zero at $I=19.8$, both Freedman (1989) and Davidge \& Nieto (1992) see significant numbers of stars brighter than $I=19.8$ extending to $I \approx 18$. Since Freedman's inner field overlaps with our own, we can test directly the hypothesis that this inconsistency is due to differences in resolution. We therefore convolved our PC image with a Gaussian kernel of FWHM $=0$ ". 6 to match Freedman's CHFT resolution. We then integer-binned the data to a scale of $0.18 \mathrm{pix}^{-1}$, reasonably close to the $0.2 \mathrm{pix}^{-1}$ scale at the prime focus of the CFHT. Finally, we scaled the counts to match the exposure times used by Freedman, adding $\sim 3$ "background" counts per pixel as well as noise to match the gain and readout noise characteristics of the RCA chip on the CFHT. To characterize the degraded PSF in the standard way, the same process was applied to the NGC 6397 observations, constructing a PSF from 27 stars still visible in the resulting image. We applied two passes of the DAOPHOT II star-detection routine and used ALLSTAR to carry out the PSF-fitting photometry. The results yielded 147 stars detected in both the F555W and F814W frames in the region of overlap between the PC field of view and that of Freedman. Of these, 68 could be matched with the photometry of Freedman, yielding a mean $I$-band magnitude difference of only $0.03 \pm 0.35$ mag. The fact that we could match only half the stars in the field we attribute to differing completeness levels, which depend on rather small differences in the noise properties.

The resulting CM diagram and luminosity function are remarkably similar to those obtained by Freedman. While the photometry of the original HST image yields only 3 stars brighter than $I=20$, smoothing the image results in a total of 19 stars measured to be brighter than this magnitude. Moreover, the mean color measured in the degraded image of the stars brighter than $I=22$ becomes bluer by 0.5 magnitudes, from $V-I=2.3$ to $V-I=1.8$. This strongly suggests that most of the bluer bright giants appearing in the ground-based CM diagrams of this region are in fact blends of fainter stars.

The existence of the infrared-luminous AGB stars found by Freedman (1992) and Elston \& Silva (1992) using $J$ and $K$ photometry appears to be compatible with our observations, although we cannot identify them uniquely with $V$ and $I$ alone. Freedman finds 125 stars with $K<18$ in a

\footnotetext{
${ }^{6}$ We use the term AGB loosely here, meaning all stars brighter than the metal-poor RGB tip. Metal rich AGB stars may be fainter than $I=20.4$, so the surface densities we derive will necessarily underestimate the true number of post-horizontal branch stars at all metallicities.
} 
$100^{\prime \prime} \times 40^{\prime \prime}$ field at similar distance from the nucleus as our HST observations; 85 of these $K$-bright stars are also detected in $J$. Given our smaller image area, we would expect to have $\sim 35$ stars with $K<18$ in our PC observations, with half of these having $K<17$. Most of these stars have $J-K>1.1$, and would thus be expected to have $I-K>3.5$ if the Milky Way bulge giants of Frogel \& Whitford (1987) can be used as a guide. We would conclude that most of the $K$-bright stars thus have $I>20$, placing them comfortably below the bright tip of our $I$-band luminosity function. The Frogel \& Whitford giants further show little correlation between $V-I$ and $I-K$ for $I-K>3.5$, so the $K$-bright stars could fall anywhere within the broad $V-I$ spread at the top of the $I$-band CM diagram. In contrast, the brightest and bluest stars in the Freedman sample might be expected to fall above the tip of the present $I$-band luminosity function; however, there are only 8 stars with $K<17$ and $J-K<1.1$ in the Freedman sample, giving an expectation of just 2 such stars for the PC field. The interesting problem posed by the Freedman (1992) and Elston \& Silva (1992) data is that the majority of their $K$-bright stars actually have $J-K$ colors much redder than the Frogel \& Whitford (1987) bulge sequence, making them presumably extremely red in $I-K$, and perhaps also $V-I$. As noted above, there are 14 stars with $I<22$ that have no $V$ counterparts - perhaps these are stars that correspond to a significant fraction of the $K$-bright sample, and for which it will be very interesting to obtain NICMOS observations.

\subsection{The Metallicity Distribution}

We argue that the composite nature of the giant branch in M32 is due to a wide range in metallicity. Gradients in the spectral index observations (see below) imply that the (light-weighted) mean age of the M32 population increases with radius, so there may well be a mixture of ages present in the PC field; however, a spread in age can account for only a modest amount of the spread in giant properties implied by the CM diagram morphology, as we now show. In Figure 0 , we show a set of three isochrones (Worthey 1994) with ages 2, 5, and 15 Gyr. Since the three tracks can be made to overlap by making only modest metallicity changes among them, the age structure is impossible to deduce from the color distribution of the giant branch alone. The tradeoff between age and metallicity for any assumed RGB track is approximately $d \log |\tau|=-1.7 d \log |Z|$, where the minus sign indicates that a younger age is counteracted by increased metallicity. On the other hand, since age effects are less important than Z, the color spread must primarily reflect the intrinsic metallicity distribution, with ambiguity in age causing only minor uncertainty. This is evident in Figure 7, where we show RGB tracks for clusters with diverse metallicities, and in Figure 8, where we show theoretical RGB tracks covering a wide range in both metallicity and age.

We can demonstrate the degree to which metallicity must predominate over age effects as follows. At $M_{I}=-1.6$, the width of the red giant branch is $\operatorname{FWHM}(V-I) \approx 0.6$ mag, giving

\footnotetext{
${ }^{7}$ Note that the behavior here is close the the behavior $d \log |\tau|=-1.5 d \log |Z|$ found for integrated colors and metal-line strengths (Worthey 1994.)
} 
$\sigma(V-I)=0.26$ mag. The photometric uncertainty at this magnitude is $\sigma(V-I)=0.14$ mag, yielding an intrinsic RGB width of $\sigma(V-I)=0.22 \mathrm{mag}$, or $\operatorname{FWHM}(V-I)=0.51 \mathrm{mag}$. Now from Figure 8 we see that $\operatorname{FWHM}(V-I)$ for $\Delta Z=1.2$ dex is $\approx 0.5 \mathrm{mag}$, giving a change in color with respect to metallicity of $0.42 \mathrm{mag} / \mathrm{dex}$. From the scaling rule above, the age dependence therefore goes as $0.42 / 1.7=0.25 \mathrm{mag} / \mathrm{dex}$. Assuming that the observed width must be the quadrature sum of both effects, we define

$$
\Delta_{t} \equiv \text { FWHM in } \log \tau
$$

and

$$
\Delta_{Z} \equiv \mathrm{FWHM} \text { in } \log Z
$$

Thus we have

$$
\left[0.42 \Delta_{Z}\right]^{2}+\left[0.24 \Delta_{\tau}\right]^{2}=0.51^{2}
$$

Setting a maximum allowable age range of $\log \tau=1.0$ dex (1.5 to 15 Gyr) and solving, we find a minimum allowable $\Delta_{Z}=1.1$ dex. The data clearly mandate a large range in metallicity irrespective of the range in stellar ages which may be present.

The CM diagram implies that the distribution of metallicity is fairly smooth, but with many more metal-rich stars than metal-poor ones. This is illustrated in Figure 9, where we compute the metallicity histogram by laying down a mesh of 10 Gyr isochrones on the CM diagram covering a wide range of $[\mathrm{Fe} / \mathrm{H}]$, and then counting the stars between them brighter than $M_{I}=-2.4$, where the uncertainties are fairly small. A small correction for differences in RGB lifetime at different metallicities was also applied. No correction was applied for $V$-band incompleteness for the reddest giants, so the highest metallicity bins slightly underestimate the true number of giants by up to 14 stars. One sees that the majority of stars lie along the red side of the RGB, with a decreasing tail toward blue, metal-poor RGB loci. There are very few stars $(<1 \%)$ more metal-poor than $[\mathrm{Fe} / \mathrm{H}] \approx-1.5$. Although some of us initially had a visual impression of bimodality in the CM diagram, with a slight paucity of stars between the main "blue" and "red" RGBs, the $[\mathrm{Fe} / \mathrm{H}]$ distribution shows no evidence of this. The illusion probably stems from the fact that RGB color begins to change rapidly with metallicity at about $[\mathrm{Fe} / \mathrm{H}]=-0.5$ and higher. The effect of assuming a population age of 15 Gyr is also shown; the histogram shifts only slightly to lower metallicity.

In Figure 9 we compare the distribution of metal abundance found in M32 with closed-box models of chemical evolution. These simple, one-parameter models are one-zone, with no gas infall or outflow and zero metal content initially, and assume instantaneous recycling of heavy elements (e.g. Searle \& Sargent 1972). The photometric uncertainties for the stars considered here are relatively small, and the models have not been smoothed. Quite striking is the fact that the 
observed metallicity distribution is significantly narrower than the closed-box models would predict. This region of M32 appears at first glance to be more monometallic than even the solar cylinder. On the other hand, if the main ridge of stars in the CM diagram is comprised of populations with a spread in age, then the youthful stars may move to the next higher $[\mathrm{Fe} / \mathrm{H}]$ bin, and it is possible that the real abundance distribution resembles that of the solar neighborhood more than it first appears in Figure 9. For example, the metal abundance of the main red component is $[\mathrm{Fe} / \mathrm{H}]=-0.2$ if one assumes an age of $15 \mathrm{Gyr},[\mathrm{Fe} / \mathrm{H}]=-0.07$ for $8 \mathrm{Gyr}$, but $[\mathrm{Fe} / \mathrm{H}]=0.01$ for $5 \mathrm{Gyr}$. Even so, the general shape of the distribution remains more or less constant with age, and the result that there are few low metallicity stars is secure.

A similar metallicity distribution derived for the POS2 M31 background field is also illustrated in Figure 9, renormalized by a factor of 5.7 so that the total number of stars is the same as that for the corrected POS1. Relative to M32, the M31 population has a peak at similar Z but a higher tail towards lower metallicities. The POS2 M31 diagram resembles the solar neighborhood more closely, with the caveat that the age structure is completely unknown. The paucity of metal-poor stars relative to the closed-box model is also inferred for the nuclei of M31 and M32 (Worthey, Dorman, \& Jones 1996), so it is probable that this condition exists at most radii in both M32 and M31.

Worthey et al. (1996) argue that the simplest explanation for the lack of low-mass, metalpoor stars is that normal processes of chemical enrichment typically operate to produce fewer low-metallicity stars than the simplest closed-box model predicts. That is, modifications to the simplest model (like variable yield, spatially inhomogeneous enrichment, or variable IMF schemes that result in fewer metal poor stars) may be required to mimic chemical enrichment in the real universe. However, it is also possible that metal-poor stars could be present at larger radii. Largeradius storage of metal poor stars is a prediction of models of monolithic galaxy collapse (e.g. Larson 1975, Matteucci \& Tornambè 1987, Arimoto \& Yoshii 1987) in which the metal poor stars are created at large radius and enrichment proceeds at smaller and smaller radii until most of the gas is consumed. The abundance distribution at a given radius predicted by these models is always narrower than the closed-box model (Larson 1975).

The predicted number of metal-poor stars in the collapse model can be expressed as a fraction of total galaxy mass. In M32, the radius of the POS1 field encloses about $75 \%$ of the total light, using the parameters of Kent (1987) and assuming a deVaucouleurs $r^{1 / 4}$ profile. Conversion to mass requires a $M / L$ ratio as a function of radius. Older ages and higher metallicities drive $M / L$ up, so most galaxies are inferred to have a higher $M / L$ in the nucleus. However, M32 is likely to be an exception to this general rule owing to the sizeable subpopulation of young stars resident in the nucleus (see Section 3.5). Various population mixtures were modeled and compared to both the nucleus and the POS1 positions, and the best-fitting $M / L_{B}$ ratios were roughly $M / L_{B}=3$ to 4.5 on a scale where Galactic globulars have $M / L_{B}=2.7$ for both M32 locations depending on the age-metallicity mixture. The amount of mass enclosed within the radius of POS1 (which we have taken to be $75 \%$ ) is consequently about $10 \%$ more uncertain than our estimate of the enclosed 
light.

In the closed-box model, about $10 \%$ of the stars in M32 should be more metal-poor than $[\mathrm{Fe} / \mathrm{H}]=-1$ assuming an approximately solar yield (the percentage would be higher if the yield is less than solar). Thus, if we stipulate a largely circular orbit distribution for metal-poor stars to account for the lack of such stars in our sample, there would still appear to be sufficient room to store them in the outer $\sim 25 \%$ of the galaxy at $R>2^{\prime}$. HST observations are scheduled for a field in the outskirts of M32 that encloses about $92 \%$ of the light. If these observations uncover copious stars of $[\mathrm{Fe} / \mathrm{H}]=-2.5$ to -1.5 , this would strongly support an outside-in dissipational origin for M32. If the observations find few such stars, then either they did not form in large numbers, as Worthey et al. suggest, or they were tidally stripped by close passages with M31, a possibility not without supporting evidence (Faber 1973; Nieto \& Prugniel 1987).

\subsection{Clump Morphology}

Turning back to the CM diagram, the morphology of the core-helium burning stars in M32 is a "clump" instead of a "blue," "extended," or "red" horizontal branch as commonly seen in Galactic (and M31) globular clusters with metallicity less than around -0.7 . This confirms the conclusion of Rose (1994) that a clump, rather than red horizontal branch stars, must dominate the core-helium burning stars to produce the observed spectral line indices and broad-band colors. A "clump" morphology is expected for metal-rich populations (Hatzidimitriou 1991), so the fact that the M32 clump is strong is consistent with the giant branch color distribution.

The extent to which the core-helium stars lie exclusively within a clump can in principle set upper limits on the numbers and ages of stars with metallicities between $[\mathrm{Fe} / \mathrm{H}]=-0.8$ and -1.5. Superficially, the CM diagram is consistent with a pure-clump population. If all core-helium burning stars are in the clump, then the metal-poor stars in M32 must be younger than 10 Gyr, as populations this young or younger are neither observed (Stryker, Da Costa, \& Mould 1985) nor predicted (e.g. Lee, Demarque, \& Zinn 1994) to have RR Lyrae stars or stars intermediate in temperature between the instability strip and the clump. This picture is contrasted against one in which most of the stars in M32 are very old. In that case the more metal-poor components should display bluer horizontal branches like the "red" one of 47 Tuc or the "extended" one of M3 (a theoretical approximation of which is shown in Figure 7 ).

Unfortunately, there are so few stars with $[\mathrm{Fe} / \mathrm{H}]<-0.7$, and the photometric errors are so large at the base of the CM diagram, that it is not possible to detect or rule out blue horizontal branch stars unambiguously. Using Figure 9 as a guide, and assuming a 15 Gyr population, there are 128 stars more metal-poor than $[\mathrm{Fe} / \mathrm{H}]=-0.7$ (out of 842 brighter than $M_{I}=-2.4$ ). The ratio of the number of bright giants corresponding to the Figure 9 cutoff to the number of horizontal branch stars in either M3 or 47 Tuc is about 0.5, so we would expect over 200 metal poor horizontal branch stars to be present under the all-15-Gyr hypothesis. To test whether we see these stars or 
not, false stars were added to the observed CM diagram in either a 47-Tuc morphology or an M3 morphology, with random errors approximately as observed. The conclusion from this exercise is that up to several hundred 47-Tuc-like HB stars could exist in the CM diagram, yet be hidden in the broad envelope of the more numerous true clump stars. Bluer M3-like HB stars could also exist, but probably less than 200 of them. In short, the present CM diagram cannot strongly constrain the upper age limits of the most metal-poor stars in M32.

\subsection{Age Information from Integrated Light}

Although the present CM diagram yields a clear picture of the metallicity distribution in M32, it does less well regarding age, especially of any younger components that might be present. Integrated colors provide some information on age if the metallicity distribution is approximately known, but the answer is intrinsically slippery because of model-to-model differences in the prediction of colors,

which translate into a 35\% scatter in age estimates (Charlot, Worthey, \& Bressan 1996). The intrinsic power of the isochrones to delimit age is also not that much higher than integrated colors: $\Delta \log Z=-1.7 \Delta \log \tau$ (see above) vs. $\Delta \log Z=-1.5 \Delta \log \tau$ (Worthey 1994). In addition, with integrated indices (including colors), we are presently limited to deriving an average age rather than a star formation history.

Worthey (1994) has demonstrated that, to break the age-Z degeneracy, it is necessary to add information beyond colors and metal lines, for example, Balmer line strength. Using this approach, we will first attempt to derive an age under the simplifying assumption that the population is characterized by a single age. G93 took long-slit spectra to obtain line-strength indices on the Lick system, which can be directly compared to the Worthey (1994) model predictions. Over the POS1 PC field of view, the G93 gradients (extrapolated from $45^{\prime \prime}$ ) were averaged, weighted by amount of light:

$$
<I>=\frac{\int_{\text {area }} I \mu_{r} \mathrm{~d} A}{\int_{\text {area }} \mu_{r} \mathrm{~d} A}
$$

where $\langle I\rangle$ is the average index, $\mu_{r}$ is the $r$-band surface brightness as a function of position from Kent (1987), and $A$ is the area over which the averaging was performed. Given the shallow color gradients in M32, $r$-band will adequately mimic $V$, where the indices are actually measured. The light-weighted mean radius of the $\mathrm{PC}$ field is $1.8 R_{e}$, where $\mathrm{G} 93 \mathrm{H} \beta=1.92, \mathrm{Mg} \mathrm{b}=2.99$, and $<\mathrm{Fe}>=2.42$. Worthey (1994) models predict that $\mathrm{H} \beta$ relative to the combined index $[\mathrm{MgFe}]=$ $(\mathrm{Mg} \mathrm{b} \times<\mathrm{Fe}>)^{1 / 2}$ can give a simultaneous estimate for a mean age and a mean metallicity, with an age in this case of $8.5 \mathrm{Gyr}$ and $[\mathrm{Fe} / \mathrm{H}]=-0.25$ for $R=1.8 R_{e}$.

This is noteworthy because if 8-Gyr isochrones are laid down on the POS1 CM diagram and a metallicity distribution computed as in Figure 9, the mean metallicity of the stars is $[\mathrm{Fe} / \mathrm{H}]=-0.25$. This demonstrates (at least) internal model coherence as shown by the close agreement in mean 
metallicity estimates from star counts in the CM diagram on the one hand vs. line strengths on the other. Within model errors, which can be substantial (Charlot, Worthey, \& Bressan 1996), the integrated colors of the M32 POS1 field are also consistent with the same age and Z.

Toward the nucleus of M32 the metallic indices of G93 get only slightly stronger, while H $\beta$ increases in strength dramatically (confirmed by Hardy et al. 1994). Differentially within the models, the only way $\mathrm{H} \beta$ and metallic indices can both increase in strength is if the mean population towards the nucleus is simultaneously younger and more metal rich. The G93 indices at the nucleus indicate a mean age of $\sim 4$ Gyr and a metallicity just less than solar. To within the uncertainties, the predicted color at the nucleus is the same as that at $1.8 R_{e}$, despite a factor of two difference in predicted age because the age effect is balanced by an average abundance enhancement.

Although age is the simplest explanation for the observed $\mathrm{H} \beta$ gradient and the flat metallic feature strength and color gradients, two other alternatives should be considered: large numbers of either blue straggler stars or blue $\mathrm{HB}$ stars, if concentrated toward the center, could increase $\mathrm{H} \beta$ strength as observed. However, both of these alternatives would make for bluer colors and weaker metallic features toward the center, which are not observed. Lacking a plausible mechanism to counter these effects, one would still require an age or metallicity gradient in M32. In the case of blue HB stars or young A-type main sequence stars, significant numbers of them appear to be ruled out in M32's nucleus by the Ca II index (Rose 1994; Worthey, Dorman, \& Jones 1996). Moreover, in contrast to M32, other elliptical galaxies generally show strongly increasing metal line strengths, reddening colors, and slightly weaker or constant $\mathrm{H} \beta$ toward the center. If blue stragglers or blue HB stars are responsible for M32's high central $\mathrm{H} \beta$, we must then explain why M32 behaves differently than other ellipticals.

Despite these favorable checks, there remains little detailed information on the age structure in M32 because all the details are averaged into one or two indicators. Dropping the assumption of a single age for the POS1 field, we constructed limited two-age models to try to quantify the uncertainty. The models begin with an assumed base age. Based on this age, a metallicity histogram is compiled from the CM diagram data. Then different age/metallicity components are substituted for the most populous bin in various proportions, and the results are compared with G93 indices. The constructed models are at all times consistent with the CM diagram for bright giants.

The (merely illustrative, not definitive) result for a base age of 15 Gyr is that about $7 \%$ (by total mass) of a 2-Gyr population is needed to match the G93 indices. Interestingly, this is consistent with the results of a near-infrared survey by Silva \& Bothun (1996) of ellipticals with strong nuclear $\mathrm{H} \beta$ and blue central colors. For a base age of $10 \mathrm{Gyr}$, 5\% of a 5-Gyr population would suffice. For a base age of $8 \mathrm{Gyr}$, the best match is obtained if there is $25 \%$ to $30 \%$ of an old, 15 Gyr population present. From the way these populations balance, it is clear that over half of the stars at the POS1 radius must be as old or older than 8 Gyr. All of these models match the data as well as the single-age 8.5 Gyr model. The conclusion is that there are many ways of balancing subcomponents so that the see-saw lands at a mean age of 8.5 Gyr. This sort of modeling, which 
takes into account CM diagram information as well as integrated light indices, is in its infancy.

\subsection{The Tip of the Red Giant Branch}

The tip of the red giant branch (TRGB) in metal-poor populations can be used as a distance indicator. Old, metal-poor giant stars have peak absolute $I$-band magnitudes of $\mathrm{M}_{I} \sim-4$ mag. Da Costa \& Armandroff (1990) calibrated the magnitude of the TRGB for the range of metallicities $(-2.2<[\mathrm{Fe} / \mathrm{H}]<-0.7)$ defined by Galactic globular clusters. With this calibration, Lee, Freedman \& Madore (1993) compared TRGB distances to 10 nearby galaxies to those from Cepheids and/or RR Lyrae stars; they found excellent correspondence at a level of $\pm 10 \%$ RMS. Subsequent studies (e.g., Sakai, Madore, \& Freedman 1996a,b) confirm the excellent agreement between the Cepheid distances and the TRGB distances for metal-poor giants. A key property of the TRGB distance indicator is that $\mathrm{M}_{I}$ varies by less than $\sim 0.1 \mathrm{mag}$ for $[\mathrm{Fe} / \mathrm{H}]<-0.7$.

Use of the TRGB distance indicator for M32 is problematic, given the high average metallicity of its population. To date, no empirical calibration for the TRGB exists for populations with metallicities higher than defined by the Galactic globular clusters. Based on the models of Worthey (1994), the expected behavior of the TRGB for more metal-rich systems is shown in Figure 10. Lee, Freedman \& Madore (1993) conclude that the method could be applied to systems as long as the galaxies show an appreciable population of low-mass, resolved red giant branch stars with [Fe/H] $<-0.7$ dex. Figures 5, 10, and 11 underscore the importance of obtaining color information before blindly applying the TRGB method. The redder, metal-rich giants in M32 are almost a magnitude fainter than the bluer, metal-poor giants, and an uncritical application of this method to the metalrich component could lead to a serious systematic error. The TRGB method works by passing an edge-detecting or "Sobel" filter over the I-band luminosity function that generates a strong signal at locations where the luminosity function has sharp discontinuities. As can be seen in Figure 11, the M32 luminosity function over all metallicities lacks a well-defined edge. Selecting just the metal poor stars (those with $V-I<1.8 \mathrm{mag}$ ) does produce a luminosity function with a sharper edge, but unfortunately in this case, there are too few such stars in the PC field to define the metal-poor TRGB accurately. Application of the edge detector to just the metal-poor giants yields a TRGB magnitude of $\mathrm{m}_{I}=20.75$, or $\mathrm{M}_{I}=-3.7 \mathrm{mag}$. This value has a large uncertainty, however, and its difference from the expected $\mathrm{M}_{I}=-4.0$ (for the assumed distance modulus of 24.43) is not significant.

\subsection{Luminosity Function}

The I-band luminosity function (LF) we derive for M32 is shown in Figure 11, along with theoretical LFs for selected age/metallicity pairs. The model LFs were generated using a pure clump, as opposed to a red or blue horizontal branch. The mismatch between the data and the 
model LFs in the clump region is likely due to a combination of $(i)$ ill-determined completeness corrections at very faint magnitudes, $(i i)$ the asymmetric nature of the photometric uncertainties in this region, which we have characterized as Gaussian for simplicity, (iii) uncertainties and a possible spread in the theoretically predicted clump luminosities, and (iv) the possibility that severe crowding is causing us to overestimate the luminosities of very faint stars in a manner analogous to that for AGB stars described in Section 3.2. Of the four model LFs shown, the $8 \mathrm{Gyr} /[\mathrm{Fe} / \mathrm{H}]=-0.07$ model is the best match to the observed discontinuity and bump in the region $20.5<I<22.0$. However, the differences among the models are sufficiently small that a slight change in the assumed distance to M32 could easily negate this distinction.

There are fairly substantial discontinuities in the LF at $I \approx 20.5$ and $I \approx 21$. The latter (which is the more significant) is clearly a consequence of the young/metal rich giant branch, which is essentially horizontal in Figure 5 at this magnitude. Assuming $(m-M)_{0}=24.43$, the discontinuity

at $I \approx 20.5$ is very close to that expected for old/metal poor stars. Indeed, if we generate a LF using only metal-poor stars bluer than $V-I=1.8$ (dashed histogram), the discontinuity is considerably enhanced. This is the tip of the giant branch used in the TRGB distance method. The cut again illustrates the importance of ensuring that the sample is not contaminated by stars more metal rich than $[\mathrm{Fe} / \mathrm{H}] \approx-0.7$ when applying the TRGB method. As we noted in the previous section, the Sobel filter actually locates the edge of the metal-poor TRGB at a slightly fainter luminosity, but with little significance.

To improve our sampling of the bright end of the luminosity function, we also analyzed the less-crowded half of chip WF4. Using a reduction procedure essentially identical to that used for the PC, this yielded another 20,000 stars (this field is slightly further from the center of M32). With allowances for the greater degree of undersampling and differing levels of completeness, the morphology of the CM diagram and the luminosity function obtained in the WF4 field are completely consistent with the results from the PC.

\section{Summary and Conclusions}

We have analyzed WFPC2 images of M32. Based on ALLFRAME crowded-field photometry we conclude the following:

- The observed spread in color among giant branch stars requires a substantial range of metallicity. Allowing for a large range in population age will have only a slight effect on the range of metallicity inferred.

- The metallicity distribution is smooth and strongly skewed towards metal rich stars. The peak of the metallicity histogram occurs at $-0.2<[\mathrm{Fe} / \mathrm{H}]<+0.05$ for assumed mean ages in the range $15 \mathrm{Gyr}$ to $5 \mathrm{Gyr}$ for the stellar population. A low metallicity tail extends to $[\mathrm{Fe} / \mathrm{H}] \approx-1.5$. 
- The raw metallicity distribution appears somewhat narrower than that of the solar cylinder although, if there are strong age admixtures, the distribution might resemble the local one more closely. In any case, the M32 distribution is much narrower than the closed-box model of chemical evolution. It remains an open question whether the missing metal-poor stars are absent entirely from M32 or whether they are stored at large radii.

- The M31 abundance distribution in the outer disk sampled by POS2 resembles the solar neighborhood if the stars there are mostly older than a few Gyr.

- The width of the giant branch remains significant at magnitudes well below the tip, also supporting the conclusion that the population has a large dispersion in metallicity.

- There is a strongly enhanced red clump at the bottom of the giant branch, consistent with Rose's (1994) analysis based on broad-band colors and spectral indices, as well as the metallicity distribution implied by the giant branch morphology. At the same time, we cannot rule out a small minority population of extended-red or even blue horizontal branch stars, which would be expected to be present if the most metal-poor stars in M32 are older than 10 Gyr.

- We do not see the very luminous blue AGB stars found in previous ground-based investigations. Experiments reveal that many of these stars are artifacts of image crowding. In contrast, a small population of the K-bright AGB candidates identified by Freedman (1992) and Elston \& Silva (1992) may be present in our sample but cannot be identified by their corresponding $I$ and $V$ luminosities alone.

- Whereas the metal poor giant branch extends to $I \approx-4.0$, the metal rich population reaches only to $I \approx-3.2$. This underscores the importance of obtaining color information and selecting only stars with $[\mathrm{Fe} / \mathrm{H}]<-0.7$ before applying the TRGB method to the determination of distances.

- The M32 CM diagram is consistent with the integrated line indices of González (1993), extrapolated to the same radius. Interpreted with Worthey (1994) models, the indices give a mean age of $8.5 \mathrm{Gyr}$ and a mean abundance of -0.25 dex. If 8-Gyr isochrones are overlaid on the CM diagram, the mean stellar abundance from counting stars as a function of color is also -0.25 dex.

- The González indices indicate a strong radial gradient in the mean stellar population with radius, the nucleus being much younger and somewhat more metal rich than the outer parts studied here. This is because the metallic indices are nearly flat but slightly increasing toward the center and the broad-band colors nearly constant, whereas $\mathrm{H} \beta$ increases strongly inwards.

- Two-age models are also consistent with both the CM diagram and the González (1993) indices, showing that there are many ways to mix ages and metallicities to match the observations. Depending on the age of the bulk of the stars, modest subpopulations of quite 
youthful stars are allowed. However at least half the stars must be at least as old as 8 Gyr. We are hopeful that future work will allow us to better constrain the options.

We thank David Silva for discussions concerning the K-bright AGB stars, and Shoko Sakai for discussions concerning detection of the TRGB. This research was conducted by the WF/PC Investigation Definition Team, supported in part by NASA Grant No. NAS5-1661. One of us (GW) is partially supported by NASA funds through grant HF-1066.01-94A from the Space Telescope Science Institute. 


\section{REFERENCES}

Ajhar, E. A., Grillmair, C. J., Lauer, T. R., Baum, W. A., Faber, S. M., Holtzman, J. A., Lynds, C. R., \& O’Neil, E. Jr. 1996, AJ, 111, 1110

Arimoto, N., \& Yoshii, Y. 1987, A\&A, 173, 23

Baum, W. A. 1959, PASP, 71, 106

Bica, E., Alloin, D., \& Schmidt, A. A. 1990, å, 228, 23

Bressan, A., Chiosi, C., \& Fagotto, F. 1994, ApJS, 94, 63

Bressan, A., Chiosi, C., \& Tantalo, R. 1996, å, in press

Burstein, D., \& Heiles, C. 1982, AJ, 87, 1165

Burstein, D., Faber, S. M., Gaskell, C. M., \& Krumm, N. 1984, ApJ, 287, 586

Buzzoni, A. 1995, ApJS, 98, 69

Charlot, S., Worthey, G., \& Bressan, A. 1996, ApJ, 457, 625

DaCosta, G. S., \& Armandroff, T. E. 1990, AJ, 100, 162

Davidge, T. J., \& Nieto, J.-L 1992, ApJ, 391, L13

Davidge, T. J., \& Jones, J. H. 1992, AJ, 104, 1365

deVaucouleurs, G. 1958, ApJ, 128, 465

Elston, R., \& Silva, D. R. 1992, AJ, 104, 1360

Faber, S. M. 1972, A\&A, 20, 361

Faber, S. M. 1973, ApJ, 279, 423

Faber, S. M., Worthey, G., \& González, J. J. 1992, in The Stellar Populations of Galaxies: Proceedings of IAU Symposium 149, ed. B. Barbuy, Kluwer Acedemic Publishers, Dordrecht, p. 255

Freedman, W. L. 1989, AJ, 98, 1285

Freedman, W. L., 1992, AJ, 104, 1349

Frogel, J. A., \& Whitford, A. E. 1987, ApJ, 320, 199

González, J. 1993, PhD Thesis, Univ. California, Santa Cruz, G93

Hardy, E., Couture, J., Couture, C., \& Joncas, G. 1994, AJ, 107, 195

Hatzidimitriou, D. 1991, MNRAS, 251545

Hodge, P., \& Kennicutt, R.C. 1982, AJ, 87, 264

Holtzman, J. A., Burrows, C. J., Casertano, S., Hester, J. J., Trauger, J. T., Watson, A. M., \& Worthey, G. 1995, PASP, 107, 1065

Kent, S. M. 1987, AJ, 94, 306 
Larson, R. B. 1975, MNRAS, 173, 671

Lee, M. G., Freedman, W. L. \& Madore, B. F., 1993, ApJ, 417, 553

Lee, Y.-W., Demarque, P., Zinn, R. 1994, ApJ, 350, 155

Matteucci, F., \& Tornambè, A. 1987, A\&A, 185, 51

Nieto, J. -L., \& Prugniel, P. 1987, A\&A, 186, 30

O’Connell, R. W. 1980, ApJ, 236, 430

Rose, J. A. 1985, AJ, 90, 1927

Rose, J. A. 1994, AJ, 107, 206

Sakai, S., Madore, B. F., \& Freedman 1996a, AJ, in press

Sakai, S., Madore, B. F., \& Freedman 1996b, AJ, in preparation

Searle, L., \& Sargent, W. L. W., 1972, ApJ, 173, 25

Silva, D. R., \& Bothun, G. 1996, in preparation

Stetson, P. 1987, PASP, 99, 191

Stetson, P. B. 1994, PASP, 106, 250

Stryker, L. L., Da Costa, G. S., \& Mould, J. R. 1985, ApJ, 298, 544

Tantalo, R., Chiosi, C., Bressan, A., \& Fagotto, F. 1996, å, in press

Trager, S. C., Faber, S. M., Burstein, D., \& Worthey, G. 1996, in preparation

Walterbos, R., \& Kennicutt, R. C. 1988, A\&A, 198, 61

Worthey, G. 1993, ApJ, 415, L91

Worthey, G. 1994, ApJS, 95, 107

Worthey, G., Faber, S. M., \& González, J. J., 1992, ApJ, 398, 69

Worthey, G., Dorman, B., \& Jones, L. A. 1996, AJ, in press 
Fig. 1.- Digitized Sky Survey image of the field containing M32. The outlines indicate the WFPC2 POS1 and POS2 fields and the Canada-France-Hawaii telescope CCD frame examined by Freedman (1989). The field shown in Figure 2 is indicated by the heavy white line. The entire field shown here subtends $15^{\prime}$ on a side.

Fig. 2.- Linear stretch of the F $814 \mathrm{~W}$ PC frame. The lower-left corner of the field is 56 arcseconds from the center of M32.

Fig. 3.- Completeness fraction as a function of $I$ and $V$ magnitudes sampled at 4 different colors. Filled symbols and solid lines correspond to the POS1 field, while open symbols and dotted lines derive from completeness tests in POS2.

Fig. 4.- Colors and magnitudes of artificial stars returned in the course of completeness tests. The open circles indicate the grid of input colors and magnitudes, and the points show the colors and magnitudes returned after processing by DAOPHOT/ALLFRAME.

Fig. 5.- ALLFRAME color-magnitude distribution of stars in the POS1 (M32) field, corrected for background contamination using the color-magnitude distribution found in the POS2 field. The magnitudes and colors shown have been transformed to $V$ and $I$ using a slightly altered transformation (zero color terms for the very reddest stars) from that given by Holtzman et al. (1995).

Fig. 6. - ALLFRAME color-magnitude distribution of stars in the POS2 (background) field.

Fig. 7.- The corrected color-magnitude diagram of stars in M32 as in Figure 5. Approximate V-band cutoffs are shown for these HST data (at $V \approx 26.0$ ) and for the Freedman (1989) data (at $V \approx 23.2$ ). Giant branch fiducials from Da Costa \& Armandroff (1990) are shown for Galactic globular clusters M15 $([\mathrm{Fe} / \mathrm{H}] \approx-2.2)$, NGC $6752([\mathrm{Fe} / \mathrm{H}] \approx-1.5)$, and 47 Tuc $([\mathrm{Fe} / \mathrm{H}] \approx-0.8)$. Also shown is the giant branch of the old open cluster NGC $6791([\mathrm{Fe} / \mathrm{H}] \approx+0.2$, age $\approx 8$ Gyr from Garnavich et al. (1994)). The reddest point on this fiducial represents the reddest giant in the cluster, but it may not correspond to the helium flash point because the RGB is sparsely populated. Three Worthey (1994) isochrones are superimposed to illustrate the degeneracy of age and metallicity in RGB color. The solid line is (age, $[\mathrm{Fe} / \mathrm{H}])=(15,-0.30)$, dotted $(5,-0.06)$, dashed $(2,+0.15)$. The predicted location of a metal-poor extended horizontal branch is shown. The line at $M_{I}=-2.4$ is the cut above which stars were counted for the histograms of Fig. 9. The error bars are from artificial star tests and refer only to color $V-I=1.34$.

Fig. 8.- Worthey (1994) isochrones are shown on the POS1 data for $[\mathrm{Fe} / \mathrm{H}]$ between -1.2 and 0.0 in 0.2-dex steps. More metal-rich isochrones are redder. Isochrones aged 8 Gyr are shown as dotted lines; isochrones aged 15 Gyr are shown as solid lines. The hypothetical locations of metal poor, extended horizontal branches are shown for both ages, the younger one nearly 0.2 mag brighter. Two examples of how bright AGB stars are predicted to lie in this diagram are shown extending beyond the 15-Gyr RGB isochrones of $[\mathrm{Fe} / \mathrm{H}]=-0.6$ and -0.4 . The errors are for color $V-I=1.34$ 
as in Figure 7 .

Fig. 9.- Counts of M32 stars caught between 10 Gyr isochrones of 1 different abundance. Stars brighter than $M_{I}=-2.4$ have small enough errors and the color change with abundance is large enough to make this a reliable method of estimating the abundance distribution of stars in M32. The raw counts between isochrones spaced at 0.2 dex intervals is shown as the coarse histogram with the heavy line. A small correction to compensate for the expected variable number of giants as a function of metallicity was incorporated. The light-line histogram assumes 15 Gyr isochrones rather than 10 Gyr isochrones. The dashed-line histogram shows the distribution for POS2 and 10 Gyr isochrones, and the counts have been multiplied by 5.7 to normalize the total number of counts to be same as for POS1. For comparison, two closed-box models of yield $\log \left[Z_{\text {yield }} / Z_{\odot}\right]=-0.5$ and -0.2 are shown. The M32 distribution is much narrower than the closed-box models. Two derivations of the abundance distribution in the solar cylinder are also shown (Rana 1991, Pagel 1989). The M32 histogram shown is even more narrow than the solar cylinder distribution, but if M32 contains many stars which are substantially younger than 10 Gyr, they will have higher abundance, and should be plotted (at most) one bin to the right. The true abundance distribution may therefore resemble that of the solar neighborhood quite closely, except for near $[\mathrm{Fe} / \mathrm{H}]=-0.5$, where M32 definitely has fewer stars than the solar neighborhood. The M32 sample of stars is 7 times larger than the sample used for the solar neighborhood studies.

Fig. 10. - Absolute I-magnitude of the tip of the red giant branch as a function of metallicity, from the models of Worthey (1994).

Fig. 11.- Background-corrected I-band luminosity function of the POS1 field before (solid histogram) and after (filled circles) applying completeness corrections. The error bars reflect counting statistics only. The theoretical luminosity functions are shown after convolution with the estimated photometric uncertainties and application of the completeness function. The 15 Gyr models are shown as solid curves while the 8 Gyr models are shown as dotted curves. The models have been normalized using the number of stars found with $22.25<I<22.5$. The dashed histogram shows the effect of counting only those stars with $V-I<1.8$. 


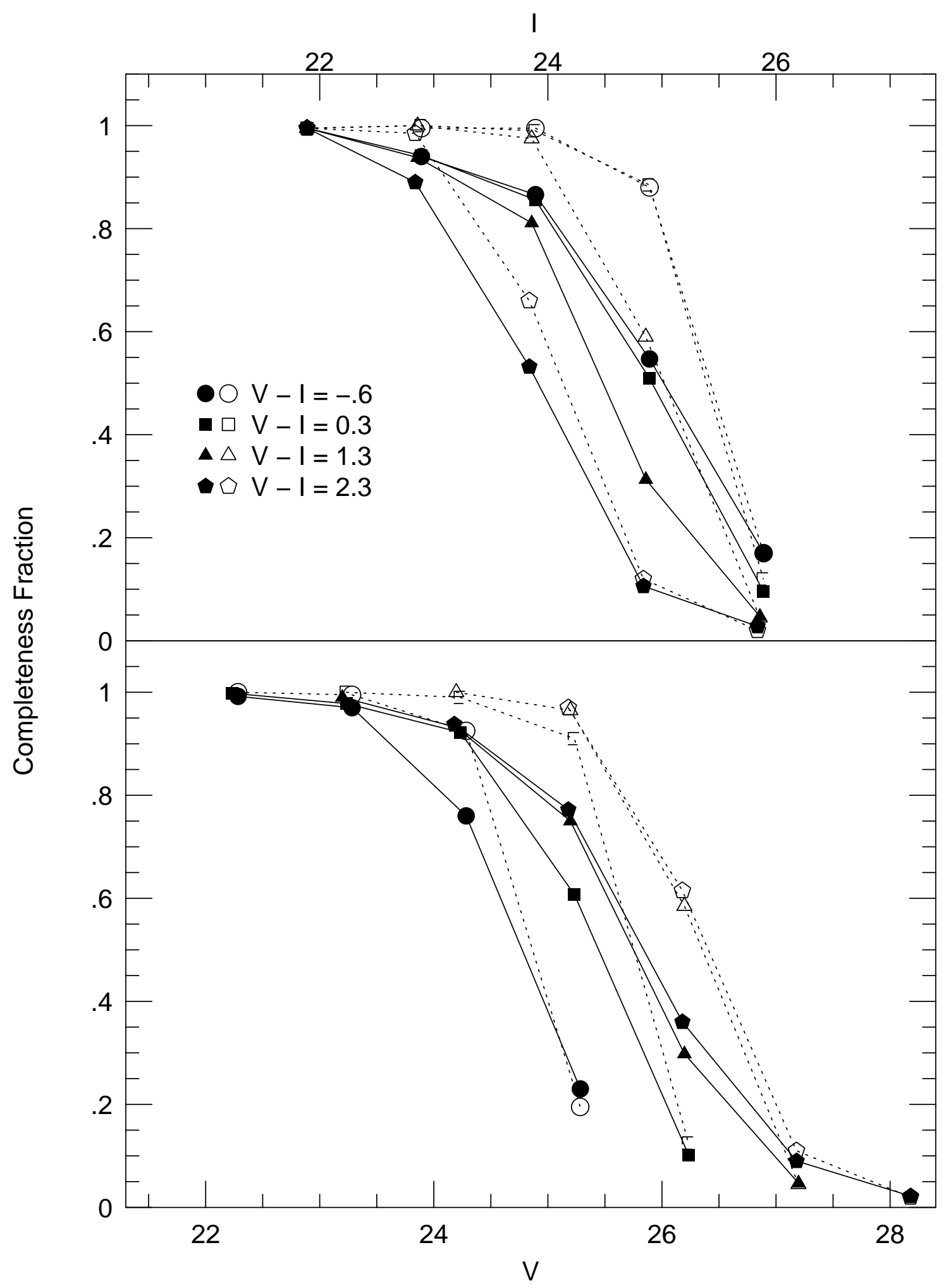

Grillmair et al. -- Figure 3 


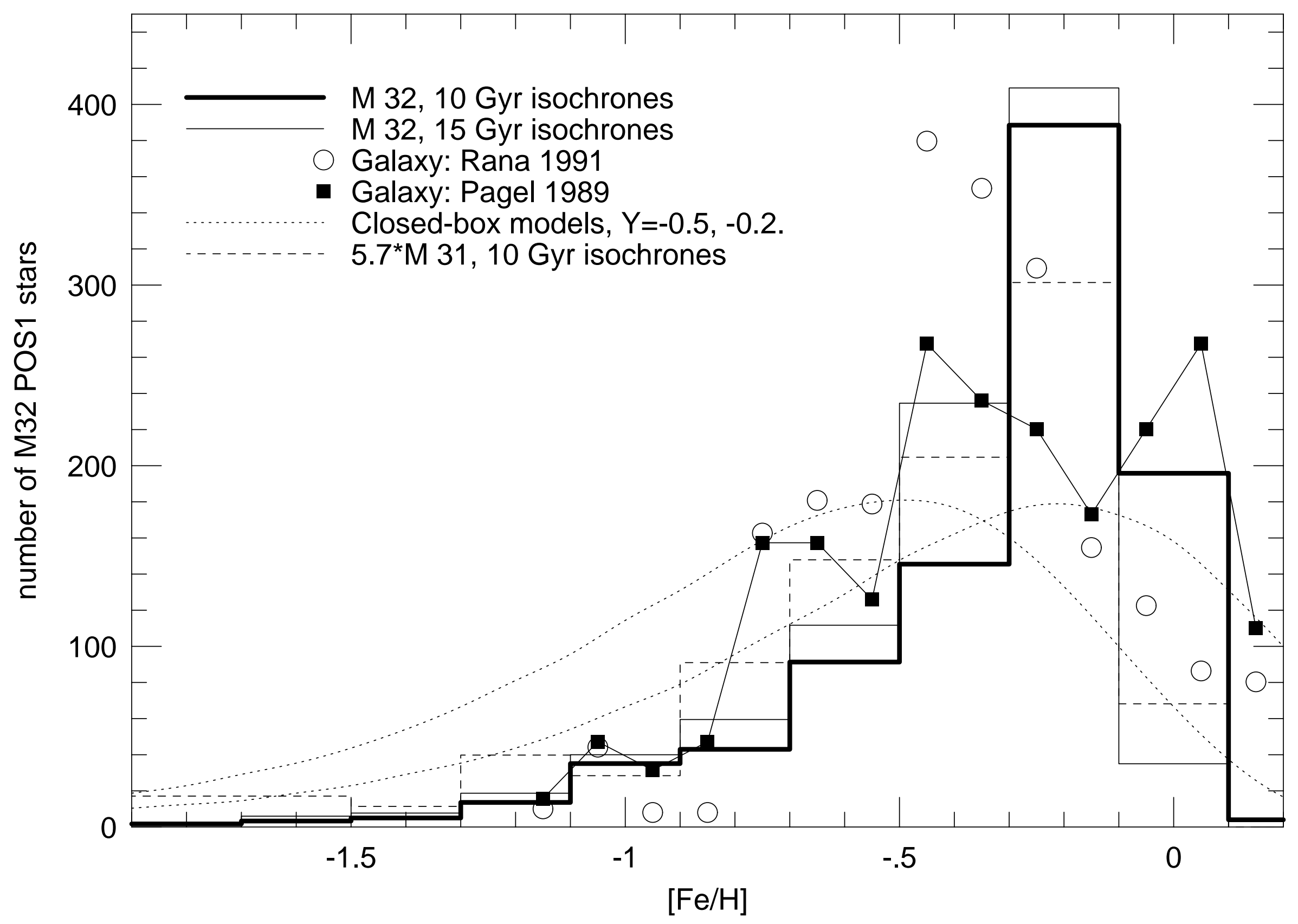

Grillmair et al. -- Figure 9 


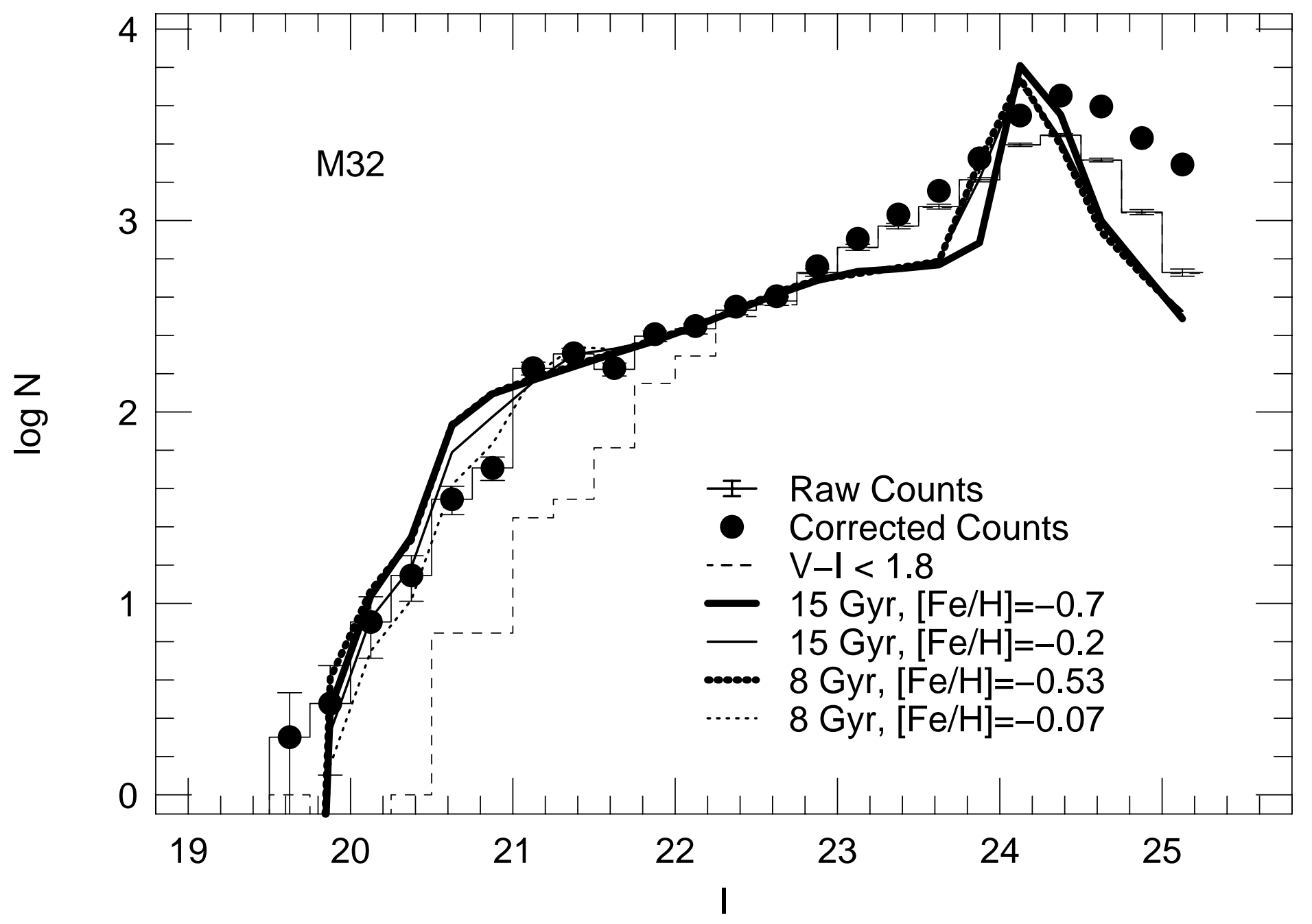

Grillmair et al. -- Figure 11 


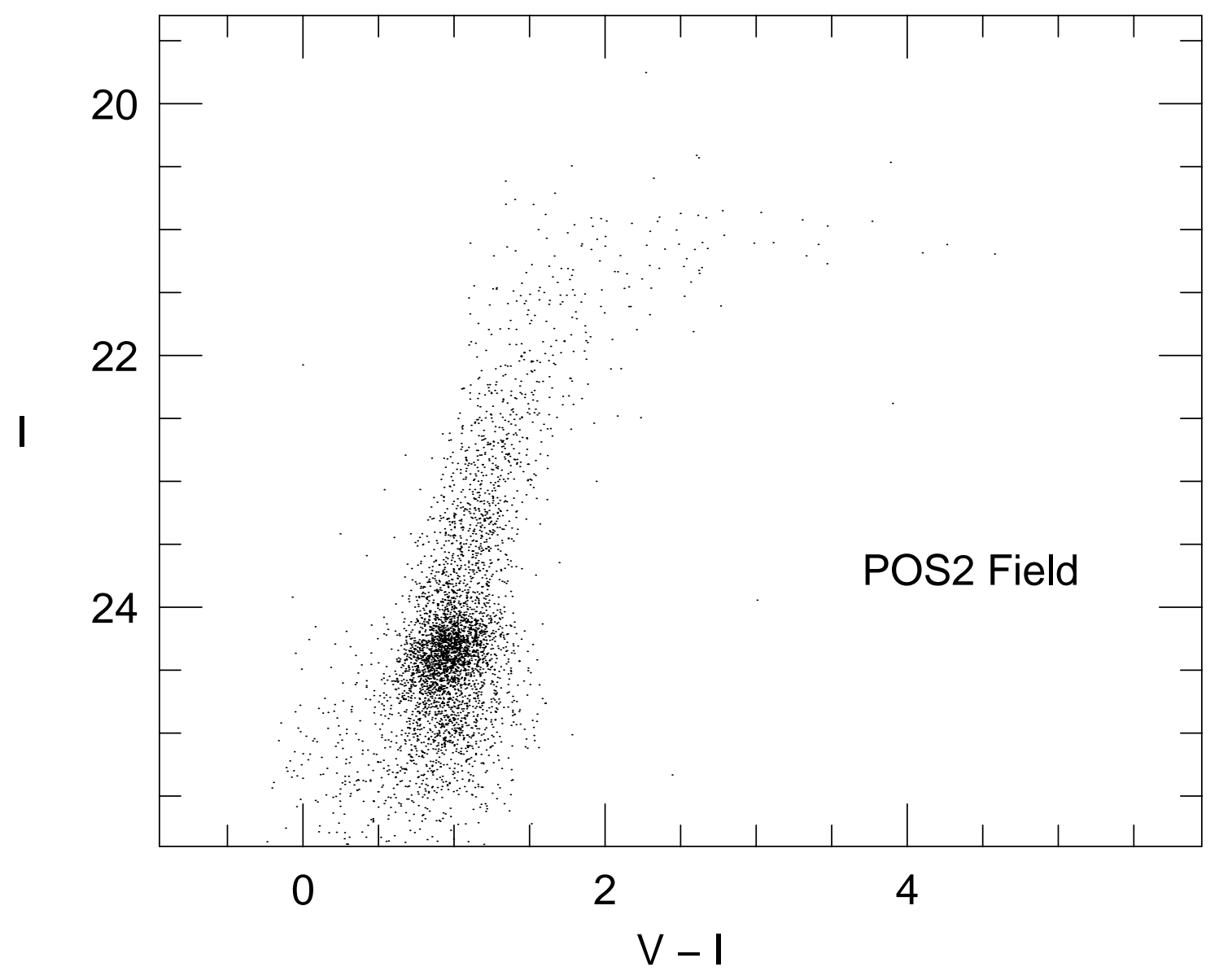

Grillmair et al. -- Figure 6 


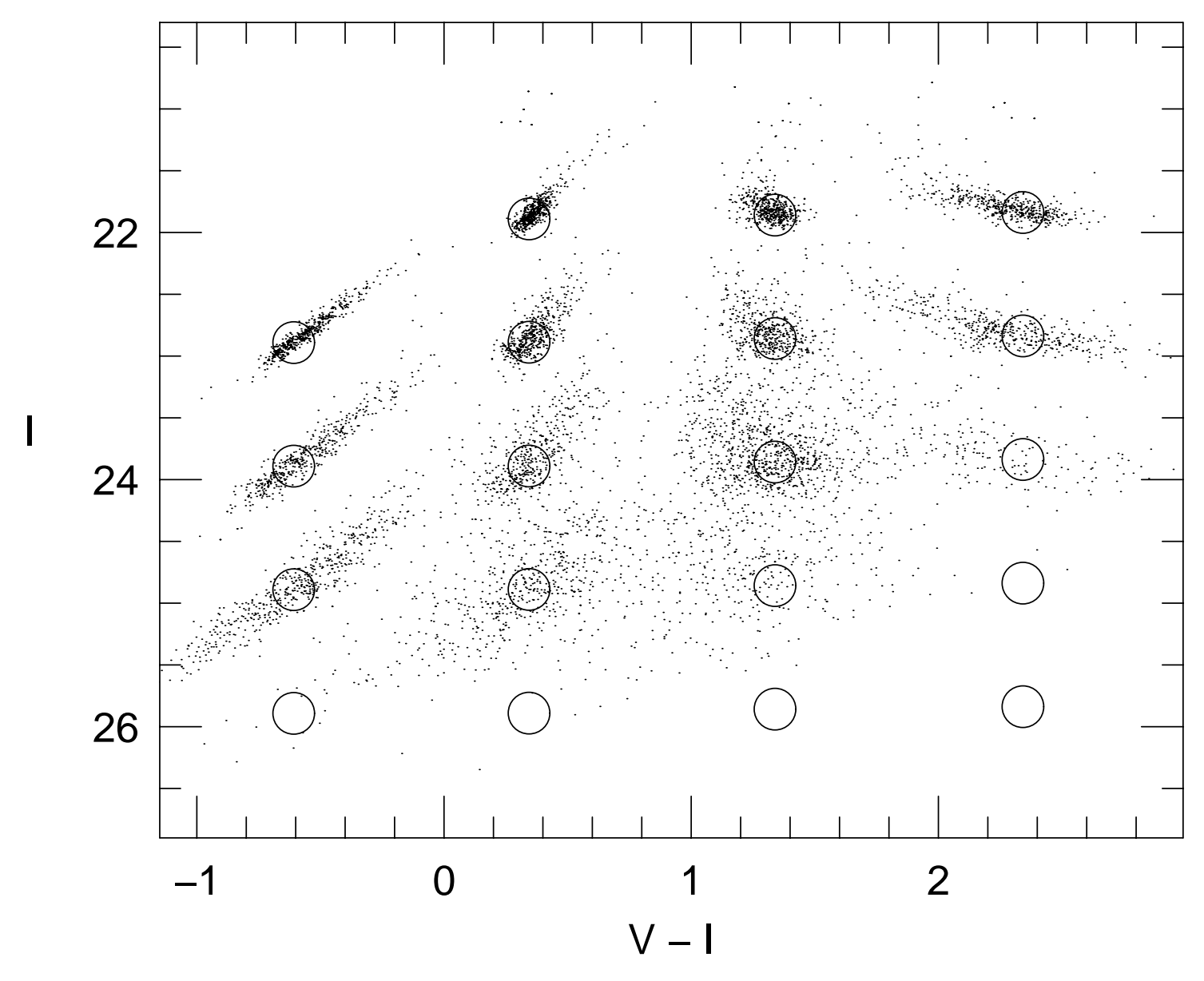

Grillmair et al. -- Figure 4 


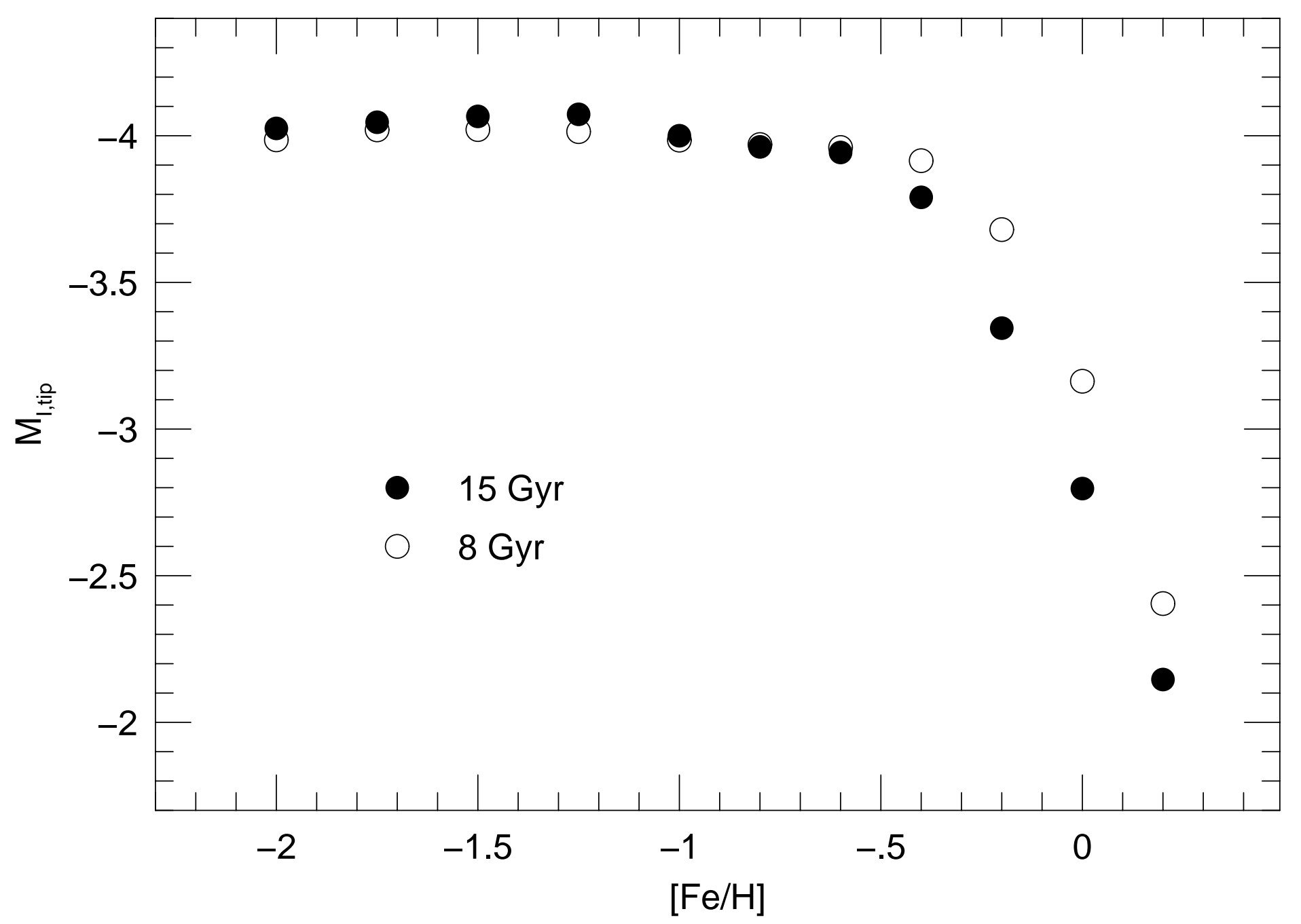

Grillmair et al. -- Figure 10 